\title{
In vitro Diagnosis of Exercise Related Skin Trauma based on Spatiotemporal Image Segmentation
}

\author{
HAOGANG CAI*
}

School of Physical Education, Shangqiu Normal University, Shangqiu 476000, China

\section{Cai et al.: In vitro Diagnosis of Exercise Related Skin Trauma}

In vitro diagnosis is a kind of product and service that can get clinical diagnosis information by detecting human samples (blood, body fluid, tissue, etc.) outside human body and then judge disease or body's functioning. This paper proposes an in vitro diagnosis method based on spatiotemporal image segmentation. Firstly, the image sequence segmentation method based on spatiotemporal Markov random field is used to segment computed tomography image, then the computed tomography image of exercise related skin trauma is segmented. According to the segmented computed tomography image, the in vitro diagnosis of exercise-related skin trauma is realized by the detection method based on the superpixel spatiotemporal characteristics. The results show that the proposed method can effectively detect skin trauma in vitro and has good segmentation effect on skin computed tomography image, as well as high accuracy in trauma detection. It can also be used in physical examination, chronic disease management and severe disease monitoring. Compared with similar detection methods, this method has significant application value.

Key words: Skin trauma, hematology, enzyme immunoassay, computed tomography

In vitro diagnosis (IVD) industry and laboratory medicine constitute an organic whole that is not only different but also closely related to each other ${ }^{[1]}$. The in vitro testing industry is the tool and weapon of laboratory medicine, while the laboratory medicine is the user and market of the in vitro testing industry. The common purpose is to implement in vitro testing ${ }^{[2]}$. About $80 \%$ of clinical diagnosis information comes from IVD and it costs less than $20 \%$ of medical expenses. IVD has become an increasingly important part of human disease prevention, diagnosis, treatment and also an increasingly important part of protecting human health and building a harmonious society. IVD has been developing rapidly in the global medical service market due to its rapid and accurate diagnosis in the early stage of the disease, playing an increasingly important role in clinical medicine and related medical research fields ${ }^{[3]}$. According to statistics, the global in vitro testing market has reached 55.4 billion United State dollars in 2013 and is expected to reach about 80 billion United State dollars in next 18 y. Developed countries such as Europe, United States and Japan are the main markets for IVD consumption. With the popularization of IVD technology and the improvement of public medical service level, IVD has been rapidly promoted in emerging countries such as China and India and achieved rapid market growth ${ }^{[4]}$.

China's IVD market started late, but grew faster ${ }^{[5]}$. From 2008 to 2012, the growth rate of China's in vitro testing market is significantly higher than the global average and the annual compound growth rate will remain at about $16 \%$. According to statistics in 2015, the market scale of IVD in China was about 28.7 billion Yuan (7.9 billion Yuan for diagnostic instruments and 20.8 billion Yuan for diagnostic reagents). Compared with the total volume of international IVD market, the domestic market share was relatively low, accounting for about $7 \%$ of the total global IVD market and the annual consumption per capita was only about 2 dollars, which was poor compared with the annual consumption per capita of about 30 dollars in developed economies. The distance is still large and IVD industry still has a large growth space in China ${ }^{[6]}$. Importantly, in the domestic IVD market the imported instruments and reagents account for more than $50 \%$ of the share, especially in the total closed detection system, the third grade

*Address for correspondence

E-mail: sqsychg@163.com 
medical institutions, etc., It occupies the main position and the future import substitution space is huge. We conservatively believe that in the next $5 \mathrm{y}$, IVD industry will still maintain a rapid growth rate of more than 15 $\%$ in China $^{[7]}$.

Objective domestic IVD is mainly concentrated in three fields: biochemical diagnostic reagent market, immunodiagnostic reagent market and hematology and flow cytometry market. Among them, biochemical diagnostic reagent market and immune diagnostic reagent market will still be the two largest markets, accounting for $60 \%$ of the total market share. Biochemical diagnostic reagents are mature both at home and abroad and have been basically replaced by domestic ones in the domestic market. Enzyme immunoassay and colloidal gold are widely used in immunodiagnosis, while chemiluminescent diagnosis is developing rapidly, occupying more and more important proportion in immunodiagnosis market. Molecular diagnostic reagent market is a potential market segment in the future and it is also a reality. The important technical premise foundation of precision medical treatment represents the cutting-edge direction of diagnosis technology and will maintain a high growth rate for quite some time in the future ${ }^{[8]}$.

At present, most of the skin trauma diagnosis is based on hospital detection and there are few research materials related to the IVD method of sports skin trauma. Wang et al. have proposed a skin diagnosis method that integrates colour and texture information, making good use of other information in image space, such as texture information and the influence of light conditions on image colour. On the basis of scanning probe microscope (SPM) method, the improvement is put forward. Firstly, the gray world algorithm is introduced to correct the colour change caused by nonstandard light, which effectively improves the positive detection rate. However, this method mainly focuses on skin colour and is not suitable for skin trauma diagnosis.

Chen et al. put forward a comprehensive skin diagnosis method based on multi features. Firstly, the image is divided into $8 \times 8$ blocks and three texture features are extracted from each block to express the texture in three directions: horizontal, vertical and diagonal. After a texture feature model judgment based on Bayesian method, the skin block is recognized. Then, two colour features of 64 pixels in the block are extracted and the skin pixels in the block are identified by the positive and negative look-up table method;
Malwin Minsky put forward skin computed tomography (CT). At present, most of the commonly used skin trauma diagnosis in hospitals are based on naked eyes or CT images. However, with the progress of the times, skin $\mathrm{CT}$ also needs to cooperate with intelligent methods to achieve intelligent skin trauma IVD. According to the research content of this paper, this paper takes skin CT image as an example, proposes an IVD method based on spatiotemporal image segmentation and innovates the detection method in vitro.

\section{MATERIALS AND METHODS}

\section{Image sequence segmentation method based on spatiotemporal Markov random field:}

This paper proposes an image sequence segmentation algorithm based on spatiotemporal Markov model (MRF), which extends the two-dimensional segmentation of exercise-related skin trauma CT image to 2.5 dimensional segmentation. It uses the information continuity between slices of exercise related skin trauma $\mathrm{CT}$ image to segment slices one by one and finally achieves the segmentation of threedimensional sequence image of exercise-related skin trauma CT image ${ }^{[9]}$.

\section{Spatiotemporal MRF model:}

According to the characteristics of spatial neighborhood group set of skin CT image, this paper defines the spatiotemporal neighborhood system of exerciserelated skin trauma CT image as shown in fig. 1. Fig. 1 shows the spatiotemporal neighborhood of slice image (layer k), slice image (layer k-1) of upper layer and slice image (layer $\mathrm{k}+1$ ) of lower layer of current exerciserelated skin trauma CT image.

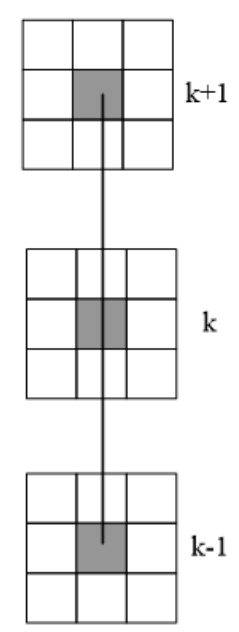

Fig. 1: Spatiotemporal neighborhood system 
In order to simplify the model, only the second-order neighborhood system (8-neighborhood) is considered. Fig. 2 shows the specific spatiotemporal neighborhood groups in the CT image of exercise-related skin trauma and a set of spatiotemporal second-order 8-pixel neighborhood groups can be obtained. Fig. 2 shows a spatial second-order neighborhood group diagram. Fig. 2 (b) and fig. 2 (c) show the second-order past group and the future group neighborhood diagram respectively. These two groups have one more secondorder neighborhood point than the spatial group, i.e., the projection point of the current pixel point in the past observation field and the future observation field (black point in fig. 2). In this paper, we call these two points the past group projection point and the future group projection point of CT image of exercise -related skin trauma $^{[10]}$.

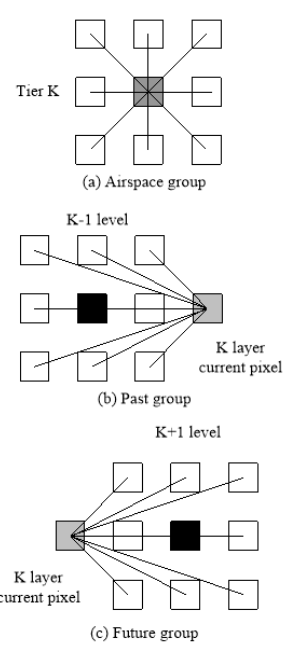

Fig. 2: Second-order 8-neighborhood groups in spatiotemporal

For point $\mathrm{s}$ on the current $\mathrm{k}^{\text {th }}$ layer image, there are 8 spatial neighbor points and 18 temporal neighbor points (fig. 3). The spatial MRF model neighborhood system only has eight neighborhood points and affects the distribution of the marker field through the energy formula in the plane. In the spatiotemporal neighborhood system, there are time domain neighborhood points. The current pixel points of the spatiotemporal image of the exercise related skin trauma CT image are not only affected by the spatial group, but also by the time domain group, which more truly reflects the moving skin trauma. There are the characteristics of continuous and gradual information between the CT images ${ }^{[1]}$.

According to this spatiotemporal neighborhood system, the neighborhood energy function of MRF can be described by Gibbs distribution. That is, the energy function $\mathrm{U}_{2}(\mathrm{w})$ defined by Markov MRF in formula (1):

Special Issue 4, 2021

$$
\mathrm{Uk}_{2}{ }_{2}(\mathrm{w})=\alpha-1 \mathrm{U}^{\mathrm{k}-1}{ }_{2}(\mathrm{w})+\alpha_{0} \mathrm{U}_{2}{ }_{2}(\mathrm{w})+\alpha 1 \mathrm{U}^{\mathrm{k}+1}{ }_{2}(\mathrm{w})
$$

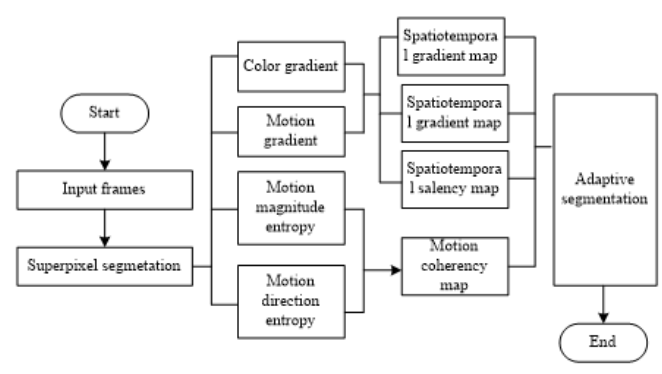

Fig. 3: Algorithm flow chart

In the formula, $\alpha_{-1}, \alpha_{0}$ and $\alpha_{1}$ are the influence factors of the potential energy functions of the past group, the spatial group and the future group in the spatiotemporal neighborhood system respectively; $\mathrm{U}^{\mathrm{k}-1}{ }_{2}(\mathrm{w})$ and $\mathrm{U}^{\mathrm{k}-1}{ }_{2}$ (w) are the potential energy functions of the current pixel in the past group, the spatial group and the future group respectively, which are defined below.

The past group diagram is shown in fig. 2 (b). Only the neighborhood group factor of two-point potential energy group is considered. There are nine past neighborhood groups in the current pixel, including the past group projection point and eight two-point potential energy groups in its eight neighborhoods. The potential energy function is expressed as:

$$
\begin{aligned}
\mathrm{U}_{2}^{\mathrm{k}-1}(\mathrm{w}) & =\sum(\mathrm{s}, \mathrm{r}) \in \mathrm{C}^{\mathrm{k}-1} \mathrm{~S} \mathrm{~V}^{\mathrm{k}-1} \mathrm{~s}, \mathrm{r}\left(\mathrm{W}^{\mathrm{k}} \mathrm{s}, \mathrm{W}^{\mathrm{k}-1} \mathrm{r}\right) \\
\mathrm{V}^{\mathrm{k}-1} 1 \mathrm{~s}, \mathrm{r} & =\left\{-\mathrm{n} \beta^{\prime} \mathrm{W}^{\mathrm{k}} \mathrm{s}=\mathrm{W}^{\mathrm{k}-1} \mathrm{r}\right. \\
\{ & \mathrm{n} \beta^{\prime} \mathrm{W}^{\mathrm{k}} \mathrm{S} \neq \mathrm{W}^{\mathrm{k}-1} \mathrm{r}
\end{aligned}
$$

Where, $\mathrm{w}^{\mathrm{k}} \mathrm{s}$ is the mark value of the current pixel point; $\mathrm{w}^{\mathrm{k}-1} \mathrm{r}$ is the mark value of the pixel point of the twopoint potential group in the past neighborhood group. When the neighborhood group $\mathrm{w}^{\mathrm{k}-1} \mathrm{r}$ is the projection point of the past group, its practical significance is that the pixel has the state of the point at the last moment. When the slice interval is very small, the changes of these two states are very small and its influence on the current pixel is stronger than its domain point, $n \geq \eta, \eta$ is an integer greater than 1 ; when the neighborhood group is the 8 neighborhood points of the projection point of the past group, then $n=1$. $\beta^{\prime}$ is the model parameter of the past group.

The definition of potential energy function of future group is similar to that of past group.

$$
\begin{aligned}
& \mathrm{U}^{\mathrm{k}+1} 2(\mathrm{w})=\sum(\mathrm{s}, \mathrm{r}) \in \mathrm{C}^{\mathrm{k}+1} \mathrm{~S} \mathrm{~V}^{\mathrm{k}+1} \mathrm{~s}, \mathrm{r}\left(\mathrm{w}^{\mathrm{k}} \mathrm{s}, \mathrm{w}^{\mathrm{k}+1} \mathrm{r}\right) \\
& \mathrm{V}^{\mathrm{k}-1} \mathrm{~s}, \mathrm{r}=\left\{-\mathrm{n} \beta^{\prime \prime} \mathrm{w}^{\mathrm{k}} \mathrm{s}=\mathrm{W}^{\mathrm{k}+1} \mathrm{r}\right. \\
& \left\{+\mathrm{n} \beta^{\prime \prime} \mathrm{w}^{\mathrm{k}} \mathrm{s} \neq \mathrm{W}^{\mathrm{k}+1} \mathrm{r} \quad(5)\right.
\end{aligned}
$$


Where, $\mathrm{w}^{\mathrm{k}} \mathrm{s}$ is the mark value of the current pixel point and $\mathrm{w}^{\mathrm{k}+1} \mathrm{r}$ is the mark value of the pixel point of twopoint potential energy group in the future neighborhood group. $\beta^{\prime \prime}$ is the model parameter of the future group. The value of parameter $n$ also depends on whether $\mathrm{w}^{\mathrm{k}+1} \mathrm{r}$ is the projection point of future group.

Due to the isotropic characteristics of skin CT image sequence, the space between the upper and lower images of the image sequence is one pixel (generally $0.128 \mathrm{~mm}$ ) and the in-plane imaging resolution of CT image can only reach one pixel size $(0.128 \mathrm{~mm} \times 0.128$ $\mathrm{mm}$ ), so the influence factor between layers is the same as that within layers. In this paper, the spatiotemporal group parameter $\beta=\beta^{\prime}=\beta^{\prime \prime}$ is set.

Then the energy function of spatiotemporal MRF is:

$\mathrm{U}(\mathrm{w} \mid \mathrm{d})=\mathrm{U}_{1}(\mathrm{~d} \mid \mathrm{w})=\mathrm{U}^{*}{ }_{2}(\mathrm{w})(6)$

\section{Description of CT image segmentation algorithm:}

By using Bayesian decision rule and maximum posterior probability estimation (MAP), we can transform the segmentation (labeling) problem of exercise-related skin trauma CT image into the maximum posterior probability estimation problem of exercise-related skin trauma CT image ${ }^{[12]}$.

$\mathrm{w}^{*}=\max _{\mathrm{w} \in \Omega}(\Omega=\mathrm{w} / \mathrm{D}=\mathrm{d})=\infty \min _{\mathrm{w} \in \Omega} \mathrm{U}(\mathrm{w} / \mathrm{d})=\min _{\mathrm{w} \in \Omega}$ $\left[\mathrm{U}_{1}(\mathrm{w} / \mathrm{d})+\mathrm{U}_{2}{ }_{2}(\mathrm{w})\right](7)$

Therefore, the problem of CT image sequence segmentation of exercise-related skin trauma can be simplified as the problem of obtaining the maximum posterior probability estimation of current pixel points under the definition of spatiotemporal $\mathrm{MRF}^{[13]}$. In this paper, the sequence of image processing is set from the CT image of exercise-related skin trauma with small label. The marker field of the past image affects the current marker field, while the future neighborhood group marker field has not been determined at this time. The future group influence factor $\alpha_{1}=0$ is set.

Due to the continuity and transmission of sequence image information, the information change between adjacent two layers is very small and the difference between the projection point of the current pixel in the past group and the current pixel is very small, so $\eta \geq 5$ is taken. By using iterated conditional mode (ICM) and information continuity between layers, the last segmented marker field information of the previous image is taken as the past group of the current image and the marker field information of the previous current image is taken as the initial segmentation of the current image, which greatly speeds up the convergence speed of CT image segmentation of exercise-related skin trauma $^{[14]}$.

\section{Algorithm step:}

The first slice of CT image of exercise-related skin trauma is segmented with spatial mixed Gaussian model Markov random field to get the marker field ${ }^{[15]}$. The information of the last CT image of exercise-related skin trauma is regarded as the past group neighborhood of the current CT image of exercise-related skin trauma and the tagging field is regarded as the initial tagging field of the current image. The tagging field with the minimum energy function is calculated by using the energy function of spatiotemporal MRF and ICM method. The parameter is $\alpha_{-1}=\alpha_{0}=0.5, \eta=0.5$.

Repeat step until the end of CT image segmentation of exercise-related skin trauma.

\section{Significance detection method of trauma based on Superpixel spatiotemporal characteristics:}

The significance detection method of trauma based on the spatiotemporal characteristics of the superpixel firstly uses the linear iterative clustering method to generate the superpixel image and reduces the feature dimension of the segmented skin CT image on the basis of preserving the integrity of the target ${ }^{[16]}$. The specific process is shown in fig. 3 .

\section{Generation of spatiotemporal gradient map:}

Considering that the edge is the most basic and effective feature to describe the spatial distribution of image, at the same time, the pixels with large optical flow changes in the time neighborhood will carry more trauma information. Therefore, on the basis of superpixel segmentation, the colour gradient feature and moving light flow feature (amplitude and direction) of single frame image are fused to represent the spatiotemporal gradient of trauma target ${ }^{[17]}$.

Let the skin CT image sequence after input segmentation be $\mathrm{I}=\left\{\mathrm{I}^{1}, \mathrm{I}^{2}, \ldots \ldots\right\}$, in the Lab colour space, the colour gradient at the pixel $\mathrm{x}_{\mathrm{i}}^{\mathrm{k}}$ of the $\mathrm{I}^{*}$-th frame is:

$\mathrm{G}_{\mathrm{c}}^{\mathrm{k}}\left(\mathrm{x}_{\mathrm{i}}^{\mathrm{k}}\right)=\left\|\left(\mathrm{L}_{\mathrm{i}}, \mathrm{a}_{\mathrm{i}}, \mathrm{b}_{\mathrm{i}}\right),\left(\mathrm{L}_{\mathrm{j}}, \mathrm{a}_{\mathrm{j}}, \mathrm{b}_{\mathrm{j}}\right)\right\|_{2}$

Where $\left\|\left(\mathrm{L}_{\mathrm{i}}, \mathrm{a}_{\mathrm{i}}, \mathrm{b}_{\mathrm{i}}\right),\left(\mathrm{L}_{\mathrm{j}}, \mathrm{a}_{\mathrm{j}}, \mathrm{b}_{\mathrm{j}}\right)\right\|$ is $\mathrm{L}_{2}$ norm. According to formula (9), the amplitude of optical flow at pixel $\mathrm{x}^{\mathrm{k}} \mathrm{i}$ in the $\mathrm{I}^{\mathrm{k}}$-th frame of the segmented skin CT image is calculated as follows:

$\mathrm{G}_{\text {om }}^{\mathrm{k}}\left(\mathrm{x}_{\mathrm{i}}^{\mathrm{k}}\right)=\sqrt{ } \mathrm{M}_{\mathrm{x}}^{2}+\mathrm{M}_{\mathrm{y}}^{2} / \max \mathrm{M}$

In the formula, $\mathrm{M}_{\mathrm{x}}{ }^{2}$ and $\mathrm{M}_{\mathrm{y}}{ }^{2}$ respectively represent the optical flow gradient in $\mathrm{x}$ and $\mathrm{y}$ direction of the 
segmented skin CT image; $\mathrm{M}=\mathrm{M}_{\mathrm{x}}{ }^{2} \mathrm{M}_{\mathrm{y}}{ }^{2} 1 ; \max (\mathrm{M})$ is the maximum amplitude, which is treated by normalization.

The optical flow direction gradient $\mathrm{G}_{\text {om }}^{\mathrm{k}}\left(\mathrm{x}_{\mathrm{i}}^{\mathrm{k}}\right)$ is defined as the average value of the difference between a certain pixel and all the pixel values in its neighborhood $\mathrm{N}_{\mathrm{s}}$ (value 8). The calculation formula is as follows:

$\mathrm{G}_{\text {od }}^{\mathrm{k}}\left(\mathrm{x}_{\mathrm{i}}^{\mathrm{k}}\right)=1 / \mathrm{N} \sum_{\mathrm{j}=1}^{\mathrm{Nj} \mid} \mid \theta^{\mathrm{k}}{ }_{\mathrm{i}}-\theta_{\mathrm{j}}^{\mathrm{k}}{ }_{\mathrm{j}} \|, \theta=\arctan \left(\mathrm{M}_{\mathrm{y}} \mid \mathrm{M}_{\mathrm{x}}\right)$

$\left\|\theta^{\mathrm{k}}{ }_{\mathrm{i}} \theta^{\mathrm{k}}{ }_{\mathrm{j}}\right\|$ represents pixels. Let the superpixel image obtained by image Ik through segmentation process be $\mathrm{Y}^{\mathrm{k}}=\left\{\mathrm{Y}_{1}^{\mathrm{k}}, \mathrm{Y}_{2}^{\mathrm{k}}, \ldots ..\right\}$. For the colour gradient graph $\mathrm{G}_{\mathrm{c}}^{\mathrm{k}}\left(\mathrm{x}_{\mathrm{i}}^{\mathrm{k}}\right)$, the boundary value of each superpixel $\mathrm{Ykn}$ is represented by the average value of the first 10 maximum pixel values in the superpixel and the superpixel boundary graph $G^{k}{ }_{c}\left(Y_{i}^{k}\right)$ is generated. The optical flow field Vk of the segmented skin CT image Ik is calculated and the optical flow amplitude and the optical flow direction gradient of each pixel in Vk are $\hat{\mathrm{G}}^{\mathrm{k}}{ }_{\text {om }}\left(\mathrm{x}_{\mathrm{i}}^{\mathrm{k}}\right)$ and $\hat{\mathrm{G}}^{\mathrm{k}}{ }_{\text {old }}$ $\left(\mathrm{x}_{\mathrm{i}}^{\mathrm{k}}\right)$ respectively. The amplitude graph $\mathrm{G}^{\mathrm{k}}{ }_{\text {om }}\left(\mathrm{Y}_{\mathrm{k}}^{\mathrm{i}}\right)$ and the direction graph $\hat{\mathrm{G}}_{\mathrm{od}}{ }^{\mathrm{k}}\left(\mathrm{Y}_{\mathrm{k}}^{\mathrm{i}}\right)$ of superpixel optical flow can be obtained. Because the characteristics of optical flow are sensitive to the interference of environment noise and camera irregular motion, under the condition of complex background texture and small difference of foreground and background colour, the superpixel boundary graph $\mathrm{G}_{\mathrm{c}}^{\mathrm{k}}\left(\mathrm{Y}_{\mathrm{i}}^{\mathrm{k}}\right)$, optical flow amplitude graph $\hat{\mathrm{G}}^{\mathrm{k}}{ }_{\text {om }}\left(\mathrm{Y}_{\mathrm{i}}^{\mathrm{k}}\right)$ and optical flow direction graph $\mathrm{G}_{\text {od }}^{\mathrm{k}}\left(\mathrm{Y}_{\mathrm{i}}^{\mathrm{k}}\right)$ are fused and a superpixel based boundary graph $\mathrm{G}^{\mathrm{k}}{ }_{\mathrm{ST}}$ with spatiotemporal information is obtained as follows:

$\mathrm{G}_{\mathrm{ST}}^{\mathrm{k}}=\hat{\mathrm{G}}_{{ }_{\mathrm{c}}}^{\mathrm{k}}\left(\mathrm{y}_{\mathrm{i}}^{\mathrm{k}}\right)_{\otimes} \hat{\mathrm{G}}_{\mathrm{om}}^{\mathrm{k}}\left(\mathrm{y}_{\mathrm{i}}^{\mathrm{k}}\right)_{\otimes} \hat{\mathrm{G}}^{\mathrm{k}}{ }_{\mathrm{od}}\left(\mathrm{y}_{\mathrm{i}}^{\mathrm{k}}\right)$

Where $\oplus$ represents the multiplication of corresponding elements in two vectors (matrices). By calculating $\mathrm{G}_{\mathrm{ST}}^{\mathrm{k}}$, we can detect the moving skin trauma area with large light flow change and colour gradient from the background.

\section{Calculation of spatiotemporal significance:}

To calculate the spatiotemporal significance, the spatiotemporal significance graph $G^{\mathrm{k}}=\left\{\mathrm{V}^{\mathrm{k}}, \mathrm{E}^{\mathrm{k}}\right\}$ corresponding to $\mathrm{Y}^{\mathrm{k}}$ of the super pixel image should be established firstly, and its topological structure is undirected weighted graph, where $\mathrm{V}^{\mathrm{k}}$ represents the set of all nodes in the segmented skin trauma CT image, $\mathrm{E}^{\mathrm{k}}$ represents the connecting line between the two nodes; the gradient weight of the connecting line $\mathrm{E}^{\mathrm{k}}$ between $\mathrm{Y}^{\mathrm{k}}{ }_{\mathrm{m}}$ and $\mathrm{Y}^{\mathrm{k}}{ }_{\mathrm{n}}$ is expressed by $\mathrm{W}^{\mathrm{k}}{ }_{\mathrm{mm}}$ and its value depends on the difference between $\mathrm{G}^{\mathrm{k}}{ }_{\mathrm{ST}}\left(\mathrm{Y}_{\mathrm{m}}{ }^{\mathrm{k}}\right)$ and $\mathrm{G}^{\mathrm{k}}{ }_{\mathrm{ST}}\left(\mathrm{Y}^{\mathrm{k}}{ }_{\mathrm{n}}\right)$ of the spatiotemporal gradient values corresponding to two adjacent superpixel s of $\mathrm{Y}^{\mathrm{k}}{ }_{\mathrm{m}}$ and $\mathrm{Y}^{\mathrm{k}}{ }_{\mathrm{n}}$, which can be expressed as:
$\mathrm{W}^{\mathrm{k}}{ }_{\mathrm{mm}}=\left\|\mathrm{G}_{\mathrm{ST}}^{\mathrm{k}}\left(\mathrm{Y}^{\mathrm{k}}{ }_{\mathrm{m}}\right)-\mathrm{G}_{\mathrm{ST}}^{\mathrm{k}}\left(\mathrm{Y}_{\mathrm{n}}^{\mathrm{k}}\right)\right\|$

In this paper, by calculating the shortest weighted geodesic distance between each superpixel and the superpixel located at the edge of the segmented CT image, the significance $\mathrm{S}_{\text {intra, }}{ }^{k}$ of the superpixel is measured, i.e, the probability of belonging to the foreground ${ }^{[18,19]}$. Heterogeneous SPCNN and its application in image segmentation. The calculation formula of $S_{\text {intra,n }}{ }^{k}$ is:

$$
\mathrm{S}_{\text {intra,n }}{ }^{\mathrm{k}}=\min _{\mathrm{vm} \in \mathrm{Yk}, \mathrm{vn} \in \mathrm{skp}} \mathrm{d}_{\text {geo }}\left(\mathrm{v}_{\mathrm{m}}, \mathrm{v}_{\mathrm{n}} \mathrm{G}^{\mathrm{k}}\right)(13)
$$

Where, $\mathrm{v}_{\mathrm{m}}$ and $\mathrm{v}_{\mathrm{n}}$ are the two nodes representing the superpixel $s$ in the spatiotemporal saliency graph $G^{\mathrm{k}}$, $\mathrm{S}_{\mathrm{p}}^{\mathrm{k}}$ is the set of all superpixel $\mathrm{s}$ along the four edges in frame $\mathrm{F}_{\mathrm{n}}^{\mathrm{k}} ; \mathrm{d}_{\text {geo }}($.$) is the geodesic distance between$ nodes $\mathrm{v}_{\mathrm{m}}$ and $\mathrm{v}_{\mathrm{n}}$ in the spatiotemporal saliency graph $\mathrm{G}^{\mathrm{k}}$, which represents the cumulative value of the shortest weighted path along the superpixel boundary. The weight of the weighted path consists of two parts, namely, the gradient weight between superpixels $\mathrm{W}_{\mathrm{mn}}{ }^{\mathrm{k}}$ and the distance weight between superpixels $\mathrm{w}_{\mathrm{mn}}(19)$

$\mathrm{w}_{\mathrm{mn}}$ can be obtained by calculating the Euclidean distance between two super pixels:

$\mathrm{W}_{\mathrm{mn}}=\left\|\mathrm{P}_{\mathrm{m}}, \mathrm{P}_{\mathrm{n}}\right\|_{2}$

Where $p_{m}$ and $p_{n}$ represent the centres of the $m$ and $n$-th super pixels respectively. After the normalization of $\mathrm{w}_{\mathrm{mn}}$, we can get the following results:

$\mathrm{w}_{\mathrm{mn}}^{\prime}=1-\exp \left(-\mathrm{w}_{\mathrm{mn}}\right)$

Then the average weighted geodesic distance $\mathrm{d}_{\text {geo }}\left(\mathrm{v}_{\mathrm{m}}, \mathrm{v}_{\mathrm{n}}, \mathrm{G}^{\mathrm{k}}\right)$ can be expressed as:

$\mathrm{d}_{\text {geo }}\left(\mathrm{v}_{\mathrm{m}}, \mathrm{v}_{\mathrm{n}} \mathrm{G}^{\mathrm{k}}\right) \min _{\mathrm{I}, \mathrm{vm}, \mathrm{vn}} 1 / \mathrm{z} \sum\left[\mathrm{w}_{\mathrm{mn} .}^{\prime} \mathrm{W}_{\mathrm{mn}}^{\mathrm{k}}\right]$

Where $\mathrm{z}$ represents the sum of all super pixels passed by the shortest path from node vm to vn in pixel Gk.

To sum up, the spatiotemporal significance $\mathrm{S}_{\text {intra,n }}{ }^{\mathrm{k}}$ of CT image $\mathrm{I}^{\mathrm{k}}$ of skin trauma after one frame segmentation can be calculated by formula (13) to formula (16) and the spatiotemporal significance map $\mathrm{P}^{\mathrm{k}[20]}$ can be obtained by normalizing the results. The spatiotemporal significant map $\mathrm{P}^{\mathrm{k}}$ is the IVD result that can judge the skin trauma caused by exercise.

\section{RESULTS AND DISCUSSION}

Fig. 4 shows the skin trauma of an athlete after falling down. Fig. 5 shows the skin CT scan results. The method in this paper is used for trauma diagnosis, and the diagnosis results are shown in fig. 6. It can be seen from fig. 6 that after the detection of this method, the cell condition of skin tissue can be observed significantly, 
the skin trauma can be judged in turn and the IVD of sports skin trauma can be realized, which verifies the effectiveness of this method. Regional comparison, the significance detection method combining background perception and colour comparison and the method in this paper. The P-R curve involves two parameters of accuracy $\mathrm{P}$ and recall $\mathrm{R}$.

As shown in fig. 7, when $\mathrm{P}$ of all methods is the minimum, the minimum $\mathrm{R}$ of this method is still higher than other algorithms. This is because the method in this paper can effectively detect the strong response area through the fusion of spatiotemporal information. It can be seen from the curve trend that the P-R curve of this method almost completely covers the curve of other methods and the optimal $\mathrm{P}$ is greater than 0.95 . And the detection efficiency also has advantages, which fully proves the effectiveness of the proposed method.
Table 1 is the comparison results of $10 \mathrm{CT}$ images diagnosed in vitro by the image segmentation method integrating region growth and graph theory, the image segmentation method based on cloud model and data field and the method in this paper. In this comparison, the segmentation accuracy of the three methods is analysed with CT image background and trauma entity image as segmentation objects. It can be seen from the data in the table that the segmentation accuracy of the proposed method is always greater than that of an image segmentation method based on region growth and graph theory and image segmentation method based on cloud model and data field. The maximum segmentation accuracy of background and entity in the skin trauma's CT image of IVD by the proposed method is 0.97 and 0.98 respectively. Then this method has the highest segmentation accuracy and the best segmentation effect.

Fig. 4: Skin trauma of an athlete after falling down

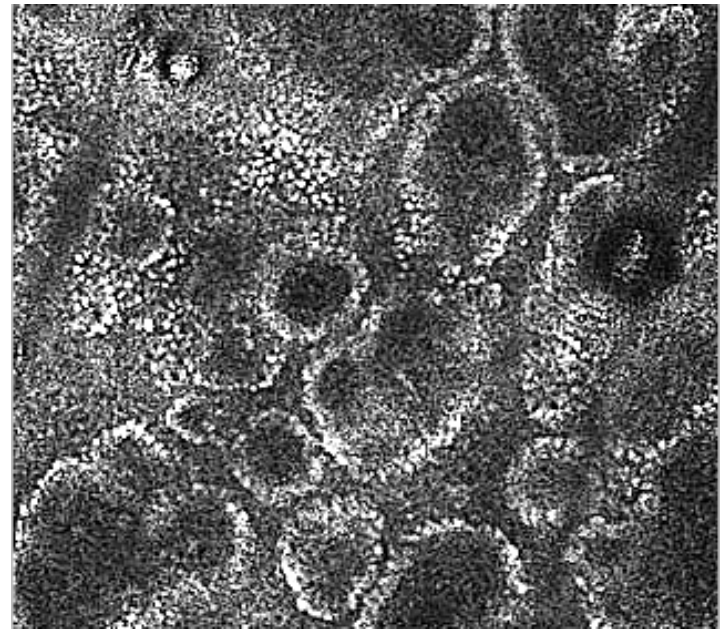

Fig. 5: CT scan results of skin 


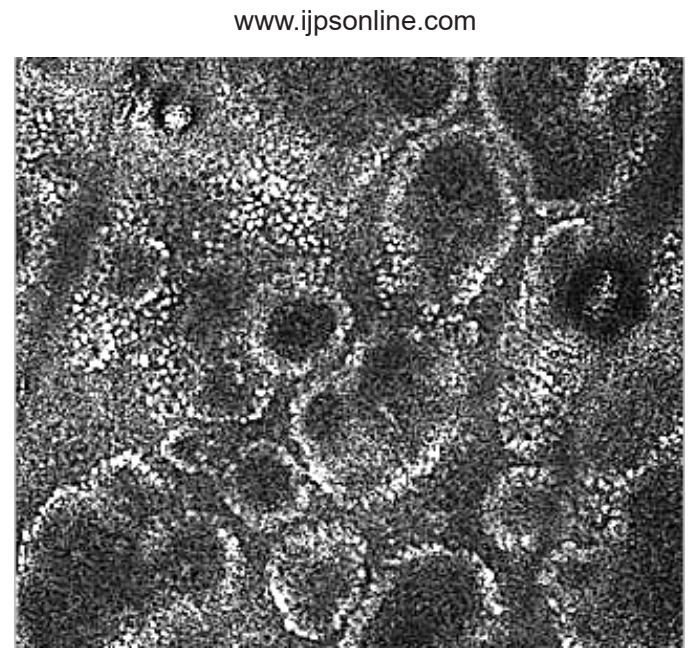

Fig. 6: Test results of this method

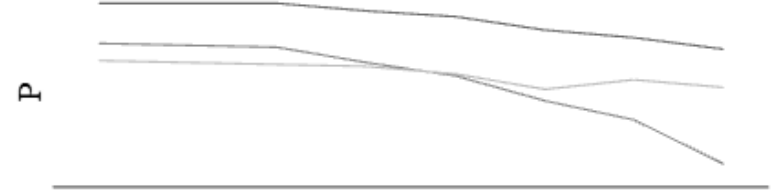

$\mathrm{R}$

Article method

Image significance detection method based on region comparison

Fig. 7: P-R curve of different significance detection methods

TABLE 1: SEGMENTATION ACCURACY OF THREE METHODS

\begin{tabular}{|c|c|c|c|c|c|c|}
\hline \multirow[t]{2}{*}{ Image encoding } & \multicolumn{2}{|c|}{ Article method } & \multicolumn{2}{|c|}{$\begin{array}{c}\text { An image segmentation method } \\
\text { based on region growth and } \\
\text { graph theory }\end{array}$} & \multicolumn{2}{|c|}{$\begin{array}{l}\text { Image segmentation method } \\
\text { based on cloud model and data } \\
\text { field }\end{array}$} \\
\hline & Background & Entity & Background & Entity & Background & Entity \\
\hline 1 & 0.96 & 0.97 & 0.91 & 0.89 & 0.82 & 0.82 \\
\hline 2 & 0.97 & 0.98 & 0.91 & 0.89 & 0.82 & 0.82 \\
\hline 3 & 0.97 & 0.97 & 0.89 & 0.88 & 0.83 & 0.81 \\
\hline 4 & 0.97 & 0.98 & 0.89 & 0.89 & 0.83 & 0.81 \\
\hline 5 & 0.97 & 0.98 & 0.89 & 0.88 & 0.82 & 0.82 \\
\hline 6 & 0.97 & 0.97 & 0.89 & 0.89 & 0.83 & 0.82 \\
\hline 7 & 0.97 & 0.98 & 0.89 & 0.88 & 0.83 & 0.81 \\
\hline 8 & 0.97 & 0.98 & 0.89 & 0.89 & 0.82 & 0.81 \\
\hline 9 & 0.97 & 0.97 & 0.89 & 0.88 & 0.83 & 0.81 \\
\hline 10 & 0.97 & 0.98 & 0.89 & 0.89 & 0.83 & 0.81 \\
\hline
\end{tabular}

In order to test the application scope of this method in depth, the proposed method, the image significance detection method based on regional comparison and the image significance detection method combining background perception and colour comparison are successively used in physical examination, chronic disease management and severe disease monitoring to describe the application limitations of the three methods with diagnosis error. The test results are shown in fig. 8, fig. 9 and fig. 10. 
www.ijpsonline.com

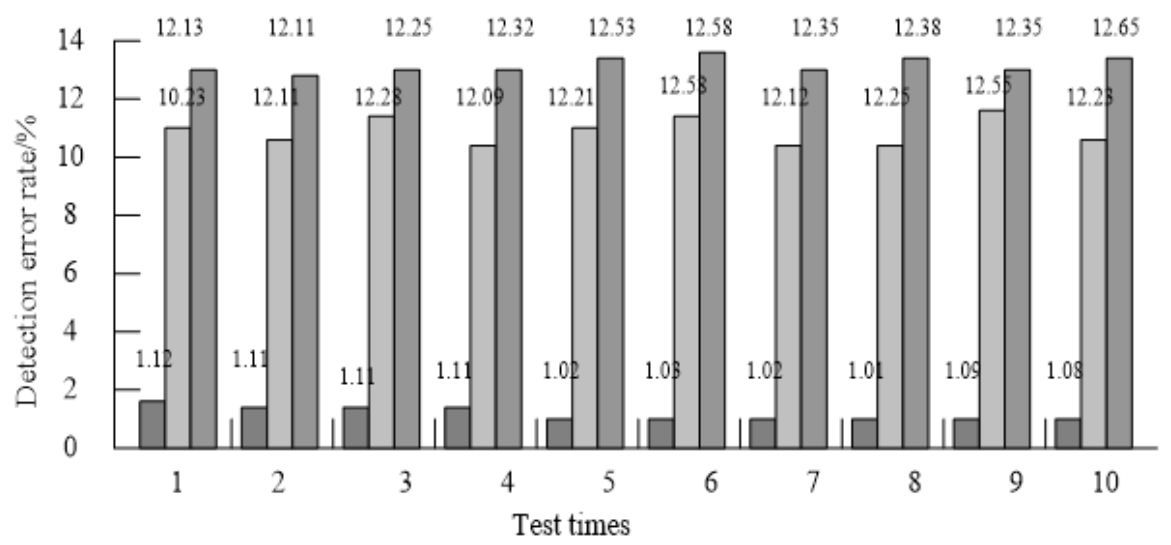

- Article method

- Image significance detection method based on region comparison

- Signficance detection method based on background perception and color contrast

Fig. 8: Examination error in physical examination

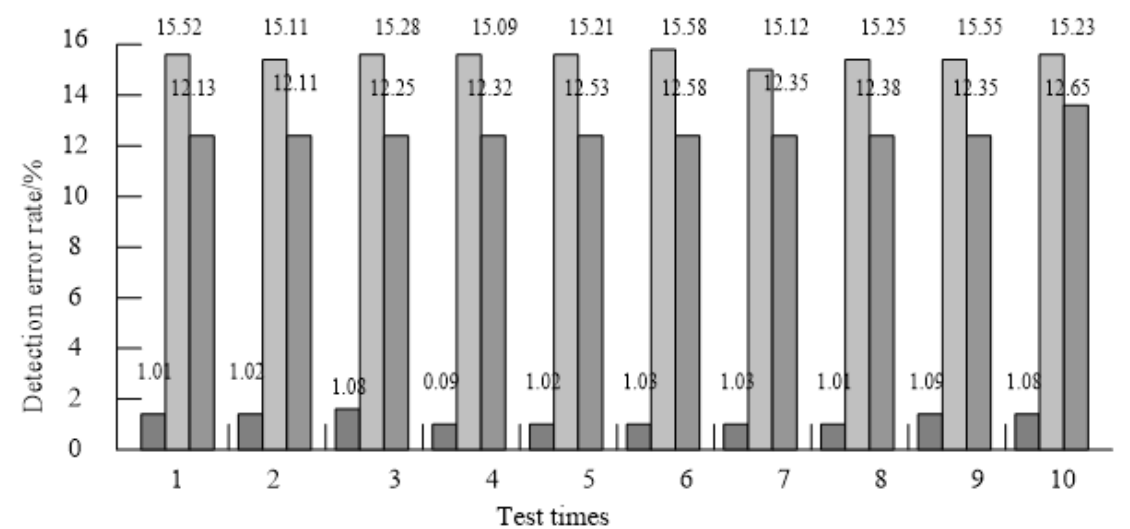

- Article method

- Image significance detection method based on region comparison

- Signficance detection method based on background perception and color contrast

Fig. 9: Detection error in chronic disease management

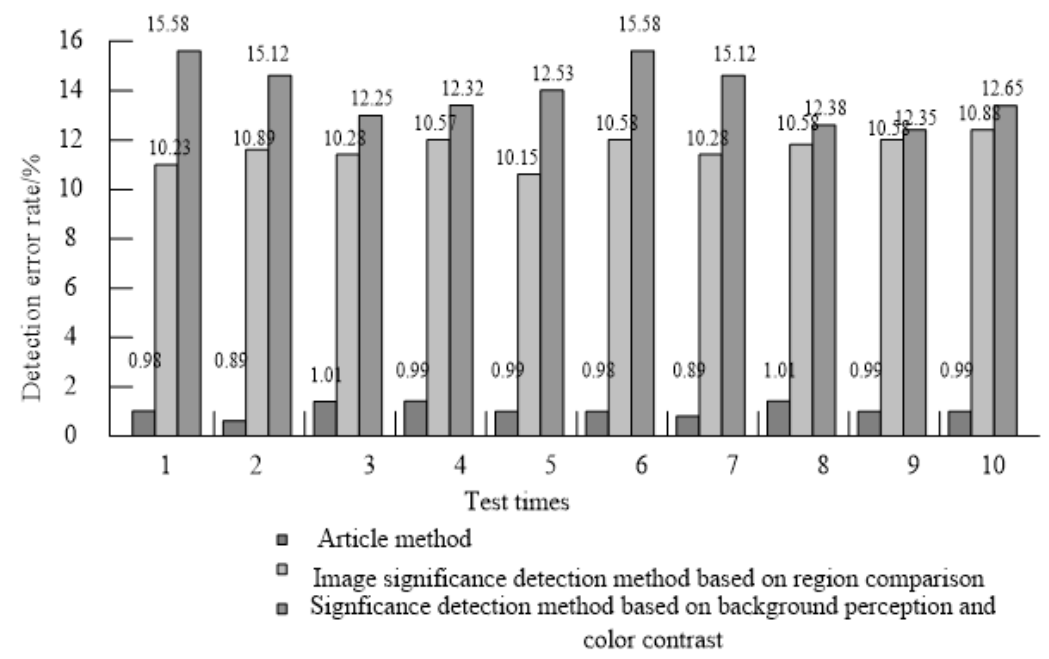

Fig. 10: Detection error in severe disease monitoring 
According to fig. 8, fig. 9 and fig. 10, the diagnosis error of the proposed method in physical examination, chronic disease management and severe disease monitoring is always the smallest and the maximum detection error of trauma in vitro in three different detection environments is $1.12 \%, 1.08 \%$ and 01.01 $\%$ respectively. The error is small, so the application limitation of the proposed method is small.

With the rapid development of IVD technology, from gene sequencing at the gene level, SNP screening, point mutation gene diagnosis, to the detection of various biomarkers at the protein level and the detection of circulating tumor cells at the cell level (CTC), thinlayer liquid-based cytology (TCT), as well as Positron Emission Tomography PET/CT at the tissue level, in general, IVD is developing in the direction of more convenient, faster, non-invasive and multi-information. The application prospects are as follows:

Modern people pay more and more attention to their health. Many people have become a habit of physical examination every year. IVD through the analysis and detection of blood, urine, feces, secretions, etc., can achieve the prevention and early detection of the disease. Worldwide, the incidence of diabetes, hypertension and chronic gastropathy is increasing year by year. There are also high incidence of hyperlipidemia, osteoporosis and other diseases. These chronic diseases need regular monitoring of blood glucose, blood pressure, blood lipid, Helicobacter pylori and bone calcium changes. Using IVD equipment, especially all kinds of wearable equipment, can monitor blood glucose, blood pressure, heart rate, etc. anytime and anywhere.

After many cancer patients receive surgery and chemotherapy, doctors can use IVD technology to regularly monitor tumor markers, predict cancer metastasis and guide medication. For example, breast cancer patients need regular chest radiographs, bone scans, liver B-ultrasound, blood routine tests and tumor related antigen tests. Researchers from British Columbia Cancer Research Center found that breast cancer patients with high expression (positive) of alphaB-crystallin gene in the brain face three times or higher risk of brain metastasis than those with low expression (negative). This allows doctors to target treatment for high-risk patients and prevent cancer metastasis.

IVD can detect human related diseases with high accuracy without harming human body. In this paper, the advantages of spatiotemporal image segmentation and IVD are combined and a method of IVD of skin trauma based on spatiotemporal image segmentation is proposed. The validity of the method is verified in the experiment and the method of IVD of skin trauma is innovated.

In this paper, an IVD method of exercise-related skin trauma based on spatiotemporal image segmentation is proposed. This method mainly uses image sequence segmentation method based on spatiotemporal MRF and trauma saliency diagnosis method based on superpixel spatiotemporal features to realize the segmentation and spatiotemporal saliency detection of skin trauma CT image. Spatiotemporal saliency detection can be understood as trauma feature extraction. It has been proved that this method can effectively integrate spatiotemporal information to detect the trauma area with strong response in $\mathrm{CT}$ image of exercise-related skin trauma and the maximum segmentation accuracy of background and entity of exercise-related skin trauma CT image IVD is 0.97 and 0.98 respectively; the P-R curve of the proposed method almost completely covers the curve of other methods, and the optimal $\mathrm{P}$ is greater than 0.95 ; the diagnosis error in physical examination, chronic disease management and severe disease monitoring is always the minimum, the maximum diagnosis error of trauma in vitro in three different detection environments is $1.12 \%, 1.08 \%$ and $01.01 \%$ respectively. Therefore, it can be seen that the application scope of proposed method is wide and compared with the comparison method in this paper, the application limitation of the proposed method is the smallest.

\section{Conflict of interests:}

The authors declared no conflicts of interest.

\section{REFERENCES}

1. Ali H, Rada L, Badshah N. Image segmentation for intensity inhomogeneity in presence of high noise. IEEE Trans Image Process 2018;27(8):3729-38.

2. Maninis KK, Pont-Tuset J, Arbeláez P, Van Gool L. Convolutional oriented boundaries: From image segmentation to high-level tasks. IEEE Trans Pattern Anal Mach Intell 2017;40(4):819-33.

3. Fan J, Wang J. A two-phase fuzzy clustering algorithm based on neurodynamic optimization with its application for PolSAR image segmentation. IEEE Trans Fuzzy Syst 2016;26(1):7283.

4. Anupama N, Kumar SS, Reddy SE. Generalized rough intuitionistic fuzzy c-means for MR brain image segmentation. Iet Image Process 2017;11(9):777-85.

5. Dehshibi MM, Sourizaei M, Fazlali M, Talaee O, Samadyar H, Shanbehzadeh J. A hybrid bio-inspired learning algorithm for image segmentation using multilevel thresholding. Multimed Tools Appl 2017;76(14):15951-86. 
6. Yan N. Resisting power attacks scheme based on signed integer splitting form. J Chin Acad Electron Info Technol 2017;12:438-42.

7. Zheng H, Liu J, Chu L. Development Status and Prospect of Multimedia Image Segmentation. Recent Pat Comput Sci 2017;10(1):62-9.

8. Sun L, Zhang Y, Chen X. Repetitive-control-based control technology for microgrid inverters. J Power Supply 2018;16:38-44.

9. Kheirkhah FM, Mohammadi HR, Shahverdi A. Modified histogram-based segmentation and adaptive distance tracking of sperm cells image sequences. Comput Methods Programs Biomed 2018;154:173-82.

10. Zhang X, Lang Y. Power management and implementation based on Ethernet switch. Chin J Power Source 2017;41:630-2.

11. Vo DM, Lee SW. Semantic image segmentation using fully convolutional neural networks with multi-scale images and multi-scale dilated convolutions. Multimed Tools Appl 2018;77(14):18689-707.

12. $\mathrm{Hu}$ Y. Main problems and solutions of mechanical and electrical equipment installation in intelligent building branch project. Autom Instrum 2017;4:159-61.

13. Liu Y, Yu J, Han Y. Understanding the effective receptive field in semantic image segmentation. Multimed Tools Appl 2018;77(17):22159-71.

14. Lin WX. Oscillation analysis for a class of even order neutral differential formulas with damping terms. J Jilin Univ 2017;55:1073-6.
15. Fang J, Liu H, Zhang L, Liu J, Liu H. Active contour driven by weighted hybrid signed pressure force for image segmentation. IEEE Access 2019;7:97492-504.

16. Ding S. Accurate evaluation and simulation of load carrying risk of building bearing column. Comput Simul 2018;35:34952.

17. Peng B, Simfukwe M, Li T. Region-based image segmentation evaluation via perceptual pooling strategies. Mach Vis Appl 2018;29(3):477-88.

18. Yang Z, Lian J, Li S, Guo Y, Qi Y, Ma Y. Heterogeneous SPCNN and its application in image segmentation. Neurocomput 2018;285:196-203.

19. Zhao XM, Li Y, Zhao QH. Self-adaptive FLICM algorithm for gray image segmentation with unknown number of clusters. Control Decis 2016;32(2):262-8.

20. Liu H, Yan M, Song E, Qian Y, Xu X, Jin R, et al. Label<? show $[\mathrm{AQ}="$ " $\mathrm{ID}=$ " $\mathrm{Q} 1]$ "? $>$ fusion method based on sparse patch representation for the brain MRI image segmentation. IET Image Process 2017;11(7):502-11.

This is an open access article distributed under the terms of the Creative Commons Attribution-NonCommercial-ShareAlike 3.0 License, which allows others to remix, tweak, and build upon the work non-commercially, as long as the author is credited and the new creations are licensed under the identical terms

This article was originally published in a special issue, "Therapeutic Perspectives in Biomedical Research and Pharmaceutical Sciences and their Nursing Methods"

Indian J Pharm Sci 2021:83(4)Spl issue "243-252" 